\title{
Should a Urinary Tract Infection Be Treated before a Total Joint Arthroplasty?
}

\author{
Javad Parvizi, MD, Kyung-Hoi Koo, MD* \\ Department of Orthopedic Surgery, Rothman Orthopaedic Institute, Philadelphia, PA, USA, \\ Department of Orthopedic Surgery, Seoul National University Bundang Hospital, \\ Seoul National University College of Medicine, Seongnam, Korea*
}

Periprosthetic joint infection (PJI) is one of the most serious complications after total joint arthroplasty (TJA). The prevalence of urinary tract infection (UTI) is common, particularly among elderly women, a group for whom TJA may be required. The association between preoperative UTI and increased risk of PJI after TJA is unclear. We reviewed key articles concerning the relationship between UTIs and PJI, and summarized recommendations of international consensus on PJI, which was established in Philadelphia in July of 2018. In addition, we distinguish between symptomatic UTI and asymptomatic bacteriuria, because their causative effects on PJI are quite different.

Key Words: Hip, Knee joint arthroplaty, Infection, Urinary tract

\section{INTRODUCTION}

A diagnosis of urinary tract infection (UTI) is made when more than $10^{5} \mathrm{CFU} / \mathrm{mL}$ is observed in urine culture $^{1)}$. Women are significantly more prone to UTIs than men; the reported prevalence of preoperative UTI in female patients undergoing total joint arthroplasty (TJA) ranges from $5.1 \%$ to $36 \%{ }^{2-7)}$.

Submitted: January 28, 2019 1st revision: February 7, 2019

Final acceptance: February 8, 2019

Address reprint request to

Kyung-Hoi Koo, MD

(https://orcid.org/0000-0001-5251-2911)

Department of Orthopedic Surgery, Seoul National University

Bundang Hospital, 82 Gumi-ro, 173 Beon-gil, Bundang-gu,

Seongnam 13620, Korea

TEL: +82-31-787-7204 FAX: +82-31-787-4056

E-mail: khkoolasnu.ac.kr

This is an Open Access article distributed under the terms of the Creative Commons Attribution Non-Commercial License (http://creativecommons. org/licenses/by-nc/4.0) which permits unrestricted non-commercial use, distribution, and reproduction in any medium, provided the original work is properly cited.
Whether preoperative UTI increases the risk of periprosthetic joint infection (PJI) after TJA is a controversial issue. According to the literature, the incidence of PJI after TJA ranges from $0.3 \%$ to $1 \%{ }^{8,9}$. Distant seeding is responsible for PJI in $10 \%$ to $20 \%$, and UTIs account for $13 \%$ of PJIs due to distant seeding ${ }^{10)}$. By this calculation, UTIs are responsible for only $0.01 \%$ to $0.03 \%$ of total PJIs.

UTIs can be associated with myriad symptoms (e.g., fever, chill, pain, leukocytosis, and pyuria), or present as asymptomatic bacteriuria (ASB). ASB presents with varying frequencies according to age, sex, and other population characteristics. In women, the prevalence of ASB increases with age, and reaches up to $20 \%{ }^{1}$. Assuming that the prevalence of ASB is 5\%, approximately 200,000 PJI patients are required to conduct a powered and highly evidenced study determining the causality of UTI for PJI, a hardly feasible scenario. Therefore, whether to treat UTI before TJA may be better determined using literatures and expert opinions.

We reviewed articles highlighting the relationship between UTIs and PJI, and summarized recommendations of an international consensus on orthopedic infections, an 
event which was held in July 2018 in Philadelphia. We separately described symptomatic UTI and ASB, because their causative effects on PJI are quite different ${ }^{3,4,6,7,11,12)}$.

\section{PREOPERATIVE SYMPTOMATIC URINARY TRACT INFECTION}

To evaluate whether UTI is a risk factor for PJI, the American Urology Association and the American Academy of Orthopedic Surgeons conducted a casecontrol study in 2003. The study included 47 cases and 200 controls and identified UTIs as an important risk factor for PJIs ${ }^{13)}$. Pulido et al. ${ }^{14)}$ prospectively observed 9,245 patients undergoing TJAs to identify risk factors of PJI, and identified preoperative UTIs as an important modifiable risk factor for PJIs; in this study, PJIs developed in 63 patients $(0.7 \%)$, and preoperative UTI was a risk factor for PJI. Yassa et al. ${ }^{12)}$ retrospectively reviewed 367 patients who underwent an emergency surgery within 24 hours for femoral neck fractures, and noted that $57(12.4 \%)$ had a surgical site infection. Among these 57 patients, $23(40.4 \%)$ had a preoperative UTI. Their study indicated that pre-operative UTI has a high prevalence among patients presenting with femoral neck fractures, and that UTI is a risk factor for surgical site infection.

On the other hand, Park et al. ${ }^{6}$ compared the incidence of PJI in a UTI group (13 patients) and non-UTI group (514 patients), and observed no significant association between preoperatively treated UTI and PJI. They treated UTI patients with antibiotics before THA if the urine culture was positive.

Thus, symptomatic preoperative UTIs should be treated before TJA. When adequately treated with appropriate antibiotics, UTI patients have similar outcomes to non-UTI patients.

\section{PREOPERATIVE ASYMPTOMATIC BACTERIURIA}

In 1984, Glynn and Sheehan ${ }^{7)}$ retrospectively analyzed 299 patients who were admitted for TJA to determine the incidence of preoperative bacteriuria and its relationship to postoperative infection. The prevalence of preoperative bacteriuria was $19.1 \%$ (57/299), and the postoperative infection rate was $3.5 \%(2 / 57)$ in patients with bacteriuria and $0 \%(0 / 242)$ in those without bacteriuria. Although there was a high incidence of
ASB, they found no correlation between ASB and PJI in these patients. Ritter and Fechtman ${ }^{11)}$ studied 364 TJAs and identified 35 cases of preoperative ASB. During the follow-up period (which ranged from 1 to 16 years), they identified 3 cases of PJI in the ASB group and 2 in the non-ASB group; none of the PJIs were related to the preoperative ASB. In both studies, there were no significantly higher infection rate in the bacteriuria group, and the microorganisms isolated from surgical site infections and urine cultures were not identical.

In 2014, Sousa et al. ${ }^{3}$ reported a higher rate of PJI in the ASB group than in the non-ASB group in a multicenter cohort study involving 2,497 patients for TJI. In their study, the prevalence of ASB was $12.1 \%$ and the overall PJI rate was $1.7 \%$. The PJI rate was higher in the ASB group than in the non-ASB group (4.3\% vs. $1.4 \%)$. Other studies including randomized clinical trial and a large cohort study showed that detection and treatment of ASB has no benefit for patients undergoing TJI. Cordero-Ampuero et al. ${ }^{4)}$ prospectively identified 45 patients with ASB from 471 patients undergoing a total hip arthroplasty or hemiarthroplasty. The 45 ASB patients were randomly assigned to receive specific antibiotics for cultured organisms (group A) or not (group B). Seven patients in group A and 6 patients in group B had wound infections after 3 months of followup. They identified no case of PJI from urinary origin in patients with ASB irrespective of whether treatment was or was not provided.

Similar findings were reported in other studies ${ }^{15-18)}$ and systematic reviews also concluded that detection and treatment of ASB has no benefit for patients undergoing TJA $^{19-21}$. All of these studies have cautioned against the adverse effects and economic burden of antibiotics.

\section{CONCLUSION}

Symptomatic UTI is a risk factor for PJI and should be treated before proceeding TJA. However, ASB is common in patients undergoing TJAs, and should not be considered as a cause for PJI. In these ASB patients, neither preoperative urine culture nor preoperative treatment is necessary before TJA.

In the 2018 International Consensus on Orthopedic Infections, the questionnaire "Symptomatic UTI must be treated with appropriate antibiotics before proceeding with the surgery. In ASB, treatment should be discontinued as it does not increase the risk of a subsequent surgical site 
infection/PJI" was agreed upon by $96 \%$ of votes, a near unanimous consensus ${ }^{22}$.

\section{CONFLICT OF INTEREST}

The authors declare that there is no potential conflict of interest relevant to this article.

\section{REFERENCES}

1. Nicolle LE, Bradley S, Colgan R, Rice JC, Schaeffer A, Hooton TM. Infectious Diseases Society of America guidelines for the diagnosis and treatment of asymptomatic bacteriuria in adults. Clin Infect Dis. 2005;40:643-54.

2.Bozic KJ, Lau E, Kurtz S, Ong K, Berry DJ. Patientrelated risk factors for postoperative mortality and periprosthetic joint infection in medicare patients undergoing TKA. Clin Orthop Relat Res. 2012;470:130-7.

3. Sousa R, Muñoz-Mahamud E, Quayle J, et al. Is asymptomatic bacteriuria a risk factor for prosthetic joint infection? Clin Infect Dis. 2014;59:41-7.

4. Cordero-Ampuero J, González-Fernández E, MartínezVélez D, Esteban J. Are antibiotics necessary in hip arthroplasty with asymptomatic bacteriuria? Seeding risk with/without treatment. Clin Orthop Relat Res. 2013;471: 3822-9.

5. Wilson MG, Kelley K, Thornhill TS. Infection as a complication of total knee-replacement arthroplasty. Risk factors and treatment in sixty-seven cases. J Bone Joint Surg Am. 1990;72:878-83.

6. Park CH, Lee YK, Koo KH. Lower urinary tract infection and periprosthetic joint infection after elective primary total hip arthroplasty. Hip Pelvis. 2017;29:30-4.

7. Glynn MK, Sheehan JM. The significance of asymptomatic bacteriuria in patients undergoing hip/knee arthroplasty. Clin Orthop Relat Res. 1984;(185):151-4.

8. Bozic KJ, Lau E, Kurtz S, et al. Patient-related risk factors for periprosthetic joint infection and postoperative mortality following total hip arthroplasty in Medicare patients. J Bone Joint Surg Am. 2012;94:794-800.

9. Kurtz SM, Lau E, Schmier J, Ong KL, Zhao K, Parvizi J. Infection burden for hip and knee arthroplasty in the United States. J Arthroplasty. 2008;23:984-91.

10. Maderazo EG, Judson S, Pasternak H. Late infections of total joint prostheses. A review and recommendations for prevention. Clin Orthop Relat Res. 1988;(229):131-42.
11. Ritter MA, Fechtman RW. Urinary tract sequelae: possible influence on joint infections following total joint replacement. Orthopedics. 1987;10:467-9.

12. Yassa RR, Khalfaoui MY, Veravalli K, Evans DA. Preoperative urinary tract infection: is it a risk factor for early surgical site infection with hip fracture surgery? A retrospective analysis. JRSM Open. 2017;8:205427041 6675083.

13. American Urological Association; American Academy of Orthopaedic Surgeons. Antibiotic prophylaxis for urological patients with total joint replacements. J Urol. 2003;169:1796-7.

14. Pulido L, Ghanem E, Joshi A, Purtill JJ, Parvizi J. Periprosthetic joint infection: the incidence, timing, and predisposing factors. Clin Orthop Relat Res. 2008;466: 1710-5.

15. Martínez-Vélez D, González-Fernández E, Esteban J, Cordero-Ampuero J. Prevalence of asymptomatic bacteriuria in knee arthroplasty patients and subsequent risk of prosthesis infection. Eur J Orthop Surg Traumatol. 2016;26:209-14.

16. Bouvet C, Lübbeke A, Bandi C, et al. Is there any benefit in pre-operative urinary analysis before elective total joint replacement? Bone Joint J. 2014;96-B:390-4.

17. Gou W, Chen J, Jia Y, Wang Y. Preoperative asymptomatic leucocyturia and early prosthetic joint infections in patients undergoing joint arthroplasty. J Arthroplasty. 2014;29:4736.

18. Bailin S, Noiseux N, Pottinger JM, et al. Screening patients undergoing total hip or knee arthroplasty with perioperative urinalysis and the effect of a practice change on antimicrobial use. Infect Control Hosp Epidemiol. 2017;38:281-6.

19.Zalmanovici Trestioreanu A, Lador A, Sauerbrun-Cutler MT, Leibovici L. Antibiotics for asymptomatic bacteriuria. Cochrane Database Syst Rev. 2015;4:CD009534.

20. Mayne AIW, Davies PSE, Simpson JM. Antibiotic treatment of asymptomatic bacteriuria prior to hip and knee arthroplasty; a systematic review of the literature. Surgeon. 2018;16:176-82.

21.Zhang Q, Liu L, Sun W, Gao F, Cheng L, Li Z. Research progress of asymptomatic bacteriuria before arthroplasty: a systematic review. Medicine (Baltimore). 2018;97: e9810.

22. Ares O, Arnold WV, Atilla B, et al. General assembly, prevention, host related local: proceedings of International Consensus on Orthopedic Infections. J Arthroplasty. 2019;34:S3-12. 